\title{
Differential proteomic screening and identification for non-traumatic necrotic femoral osseous tissue
}

\author{
PENG YAN ${ }^{1}$, YEPING ZHU ${ }^{2}$, HUI ZHAO ${ }^{1}$, YANYAN LU ${ }^{1}$ and YUZHONG GAO ${ }^{1}$ \\ ${ }^{1}$ Department of Orthopedics, The First Affiliated Hospital of Jinzhou Medical University; \\ ${ }^{2}$ Recovery Unit, Jinzhou Central Hospital, Jinzhou, Liaoning 121000, P.R. China
}

Received September 6, 2016; Accepted February 13, 2017

DOI: $10.3892 /$ etm.2017.4326

\begin{abstract}
Currently, there is a lack of effective early screening and detection methods for femoral head necrosis. Current research on most orthopedic diseases focuses on proteomics in the preliminary stage. The recent fluorescence differential in gel electrophoresis (DIGE) has advantages such as a high reproducibility, high sensitivity, high throughput, and high dynamic range. It is currently one of the most widely used quantitative proteomic research means. We conducted this study to investigate the pathogenesis of non-traumatic femoral head necrosis using the fluorescence DIGE to screen non-traumatic femoral head necrosis based on proteomics and provide a theoretical basis for screening possible biomarkers and molecular targeted treatment. The DIGE technique was used to separate the protein. An electrophoretogram was established on the basis of scanning and analysis. Identification and a bioinformatics analysis were conducted for the differential protein. The protein with differential expression of over 2-fold was excavated and ionized by means of substrate assisted laser desorption. The flight time was identified with a mass spectrometer (matrix-assisted laser desorption/ionization time-of-flight mass spectrometry, MALDI-TOF/TOF). The formation on sequences, structures and functions of these proteins were obtained through database retrieval. Western blot analysis was used to verify the differential protein expression and the reliability of the DIGE result was verified. DIGE was used to successfully separate $1,500 \pm 40$ protein spots. There were 252 significant differential protein spots. The Ettan ${ }^{\mathrm{TM}}$ Spot Picker automatic work station was used to excavate 49 significant differential protein spots with expression difference over 2-fold. The MALDI-TOF/TOF mass spectrometer was used to identify these differential protein spots. Six proteins were identified in total, which include
\end{abstract}

Correspondence to: Dr Yuzhong Gao, Department of Orthopedics, The First Affiliated Hospital of Jinzhou Medical University, 5-2 Renming Street, Jinzhou, Liaoning 121000, P.R. China E-mail: gaoyuzhonglnjz@163.com

Key words: femoral head necrosis, proteomics, fluorescence differential gel electrophoresis, mass spectrum, bioinformatics apolipoprotein A1 (APOA1), fibrous protein original chain, fibrous protein original chain, serum albumin, sulfur-oxygen protein peroxiredoxin 2 (PRDX2) and actin. APOA1 and PRDX2 were subject to western blot analysis detection; results were consistent with the DIGE result. Based on an analysis of the biological information, these proteins may be associated with the incidence and progression of femoral head necrosis.

\section{Introduction}

Osteonecrosis of the femoral head (ONFH) refers to the degenerative necrosis of the femoral head bone marrow hematopoietic cells, bone marrow adipocytes, and osteocytes (1). Research indicates that ischemic necrosis of the femoral head is the most common hip joint disease followed by hip joint tuberculosis (2). Femoral head necrosis is characterized by complex causes, a slow course of disease, and a high disability rate. Currently, there are a few cases where femoral head necrosis can be detected in its early stage and treated with drugs or surgeries due to the absence of effective early screening and detection methods.

The causes of traumatic femoral head necrosis are well defined but the causes and pathogenesis of non-traumatic femoral head necrosis are still controversial. Currently, it is generally accepted that hormonal and alcoholic ischemic necrosis of femoral head are the most common causes of non-traumatic femoral head necrosis $(3,4)$. Previous studies on proteins only focused on one or several types of proteins that are essential for osteonecrosis. Research, however, is not able to systematically explain the basic mechanism of osteonecrosis as a whole. Hence, it is imperative to study proteins in a comprehensive manner and on a large scale.

Proteomic research can reveal the pathogenic mechanisms behind the incidence and progression of major human diseases $(5,6)$. Many breakthroughs have been achieved in disease diagnosis and treatment (7-9). However, proteomic research on orthopedic diseases is largely in the preliminary stage. Thus, it is theoretically and practically significant to understand the type, quantity, and peak-value change regularity of related regulatory genes and functional gene protein products during the process of femoral head necrosis at the genomic and proteomic level by further studying the pathogenesis of femoral head necrosis using the proteomic technology. 
The proteomics research on the necrotic osseous tissue of femoral head may fundamentally reveal proteomic changes in the femoral head necrosis. It contributes to the proteomic exploration of the pathogenesis and provides a theoretical foundation for prevention and treatment of ischemic necrosis of the femoral head. Therefore, this study was conducted to collect a sample of the osseous tissue of the necrotic femoral head of adult patients with non-traumatic femoral head III-IV necrosis requiring replacement of artificial hip joints. The liquid nitrogen grinding physical method is used to extract the total protein of the osseous tissue. The fluorescence differential in gel electrophoresis (DIGE) (10) and matrix-assisted laser is used for desorption ionization. The flight time and flight time mass spectrometry (matrix-assisted laser desorption/ionization time-of-flight mass spectrometry, MALDI-TOF/TOF) technique (11) is used to analyze the expression of protein in the necrotic tissue, screen for biomarkers with diagnostic value and target spots of molecular targeting treatment, and explore the pathogenesis of the disease. To date, no related similar research method has been reported.

\section{Materials and methods}

Sample selection. We selected patients who underwent an artificial hip joint replacement surgery in the First Affiliated Hospital of Jinzhou Medical University from June 9, 2010 to October 27, 2010. Three patients were diagnosed with non-traumatic femoral head necrosis (average age, 69.3 years) in the Ficat W stage. The control group comprised of patients with fresh fractures of neck of femur aged 76.3 years on average including 1 garden III-type patient and 2 IV-type patients. Cause of injury was a fall. The average time from the injury to the surgery was 8 days. This study was approved by the Ethics Committee of the First Affiliated Hospital of Jinzhou Medical University. Signed written informed consents were obtained from all participants before the study.

Protein extraction. The extraction of the total protein in the osseous tissue was as follows: 3 different samples were taken from the necrosis group and the control group and mixed after the osseous grains were weighed; $0.3 \mathrm{~g}$ of the sample was placed in a mortar containing liquid nitrogen (Barui Medical Equipment Co., Beijing, China) and the osseous tissue was ground into powder. It was guaranteed that the liquid nitrogen was not completely volatile; $100 \mathrm{mg}$ of the osseous tissue was added to the $200 \mu \mathrm{l}$ protein lysis buffer and mixed by vibration. The solution was subjected to pyrolysis for $1 \mathrm{~h}$ on ice and then subjected to ultrasonication for $200 \mathrm{sec}$. The lysis solution (Biosharp, Hefei, China) was pipetted into an Eppendorf tube and centrifuged at $12,000 \mathrm{x}$ g for $30 \mathrm{~min}$ at $4^{\circ} \mathrm{C}$. The supernatant was taken as the total protein extracting solution and preserved at $-80^{\circ} \mathrm{C}$. The $2-\mathrm{D}$ clean-up kit (GE Healthcare, Piscataway, NJ, USA) was used to remove the substances that could intervene with the two-dimensional electrophoresis, such as salt, fat, and polysaccharide.

Two-dimensional fluorescence difference gel electrophoresis. The sample was diluted to $5-10 \mathrm{mg} / \mathrm{ml}$. The $\mathrm{pH}$ of the sample was set to 8.5. For $50 \mathrm{~g}$ protein, $400 \mathrm{pmol}$ stain (Cy2, Cy3, and Cy5) (BD Pharmingen, San Diego, CA, USA) was well mixed. The mixture was placed on ice for $30 \mathrm{~min}$ in the dark. The marked sample protein was loaded to the $24 \mathrm{~cm}$ non-linear IPG adhesive tape of $\mathrm{pH}$ 3.0-10.0 and placed in Ettan $^{\mathrm{TM}}$ IPGphor (Beckman Coulter, Fullerton, CA, USA) for first dimensional electrophoresis. The parameters were set as follows: $30 \mathrm{~V}, 12 \mathrm{~h} ; 100 \mathrm{~V}, 1 \mathrm{~h} ; 500 \mathrm{~V}, 1 \mathrm{~h} ; 1,000 \mathrm{~V}$, $1 \mathrm{~h} ; 3,000 \mathrm{~V}, 1$ h; 8,000 V, 3 h; 8,000 V, 3 h; 50,000 V, 1 hh. The dithiothreitol SDS equilibrium liquid (containing DTT $10 \mathrm{~g} / \mathrm{l}$ ) and iodoacetamide SDS equilibrium liquid (containing acetamide $25 \mathrm{~g} / \mathrm{l}$ ) were used for the equilibrium of the IPG adhesive tape in sequence for $15 \mathrm{~min}$, respectively, following electrophoresis. The Etta II DALT Six electrophoresis apparatus (Beckman Coulter) was used for the second dimensional electrophoresis.

It was electrophoresed for $45 \mathrm{~min}$ at $3 \mathrm{~W}$ and at constant power. Then, it was electrophoresed at $17 \mathrm{~W}$ and at constant power until bromophenol blue moved to the outside of the gel. The electrophoresed gel plate image was scanned with the Typhoon Imager 9400 (GE Healthcare). The protein spots had an average volume difference more than 2-fold, which were found to be based on DIA and BVA using DeCyder v6.5 analysis software (GE Healthcare, Buckinghamshire, UK).

Mass spectrum identification. The sample targets were dried in a clean space at room temperature. Compressed nitrogen was used on the sample surface. The sample target was entered to the ABI 4800 MALDI-TOF/TOF mass spectrometer (GE Healthcare) and corrected with an internal standard method. Each sample was bombarded 600 times with a laser (intensity, 3,000-4,000) and 30 points were selected at random. Each point was bombarded 20 times to obtain the peptide mass fingerprinting (PMF). The Mascot software version 3.1 (Mascot Soft Web Solution Pvt. Ltd., Varanasi, India) was used for database retrieval and comparison of the mass spectrum PMF. MS and MS/MS were combined to search in the NCBI non-redundant database (http//www.protein.sdu. $\mathrm{dk} / g$ pmaw/GPMAW/Databases/NCBInr/ncbinr.html), species database.

Statistical analysis. SPSS 19.0 software (IBM, Armonk, NY, USA) was used for statistical analysis, All quantitative data are expressed as mean \pm standard deviation. Comparison between groups was done using Student's t-test. $\mathrm{P}<0.05$ was considered to indicate a statistically significant difference.

\section{Results}

Protein expression profile of the two-dimensional DIGE. Clear protein expression profiles were obtained on all $\mathrm{pH} 3.10$ gradient strips following two-dimensional differential gel electrophoresis. The samples marked with different stains exhibited profiles of different colors and a large number of differential protein spots were present after DeCyder software was used for false-color treatment.

Mass spectrum identification for proteins. The ABI 4800 MALDI-TOF/TOF mass spectrometer was used to identify 50 differential protein spots, which were then subject to enzymolysis. Then, the peptide enzymatic hydrolysis PMF profiles were obtained by the mass spectrum. The MS-MS 
A

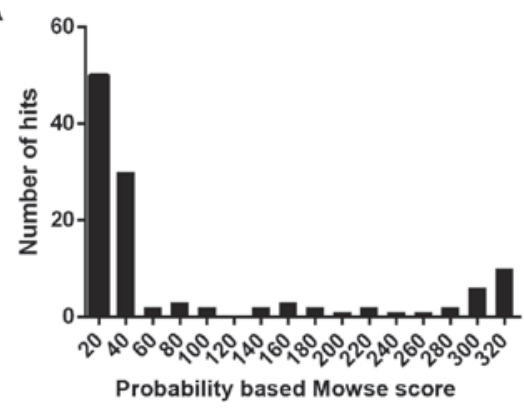

C

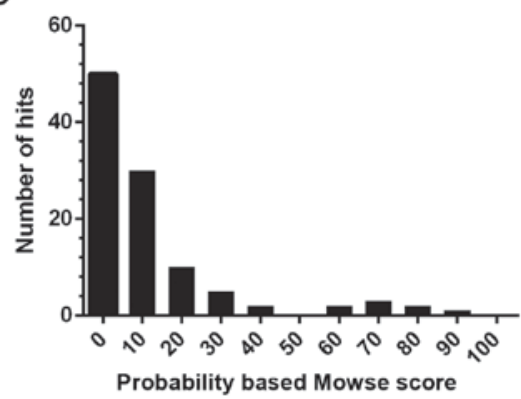

$\mathrm{E}$

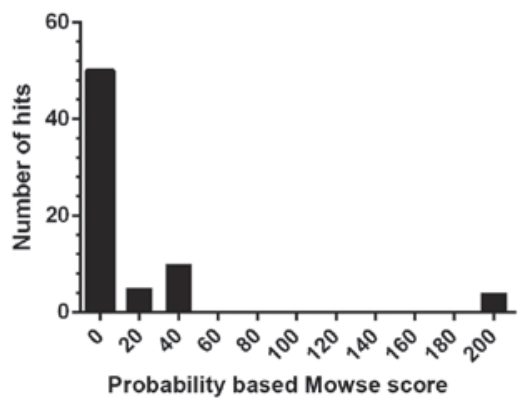

B
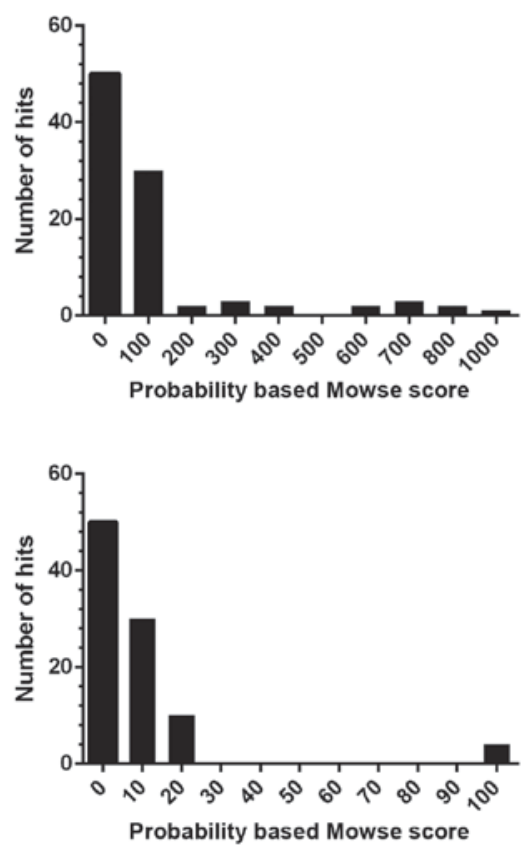

$\mathrm{F}$

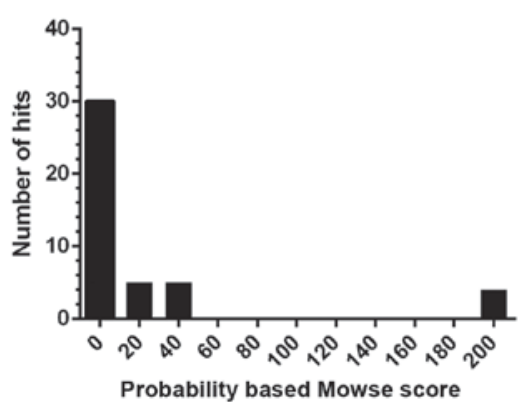

Figure 1. Mass spectrum identification Mowse score of differential protein. (A) Mowse scores of apolipoprotein A1 (APOA1), (B) peroxiredoxin 2 (PRDX2), (C) ACTG1, (D) ALB, and (E and F) FGB proteins. The score of a peptide fragment of APOA1 was 314; the score of PRDX2 was 85; ACTG had 2 adjacent peptide fragments with ALB of 480, FGB of 200, and FGG of 198. Appropriate protein types were obtained by a mass spectrometric analysis and a database comparison.

\section{A}

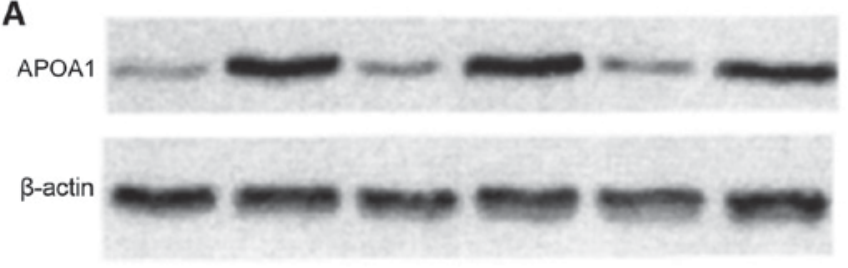

B

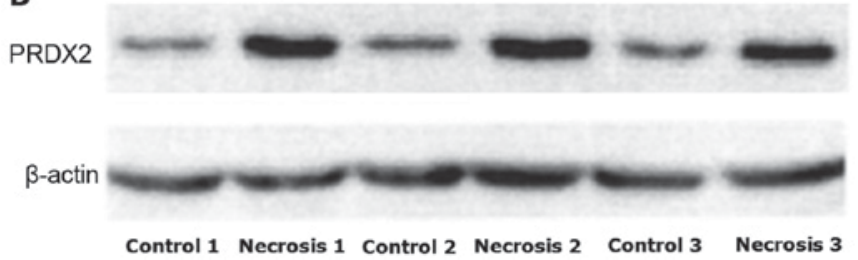

Figure 2. Protein expression levels of apolipoprotein A1 (APOA1) and peroxiredoxin 2 (PRDX2) in the necrosis group and the control group. (A) Protein expression levels of APOA1 in the control and necrosis groups (western blot analysis, $\mathrm{P}<0.05$ ). (B) Protein expression levels of PRDX2 in the control and necrosis groups (western blot analysis, $\mathrm{P}<0.05$ ) (internal reference: actin).

$1 \mathrm{kV}$-positive ion acquisition and treatment method was used. The strongest 10 parent ions (precursor) were automatically selected and subjected to MS/MS tandem mass spectrometry. The NCBI non-redundant database was searched with the combined MS and MS/MS mode for comparing PMF; the species database was a human database. The identification results with a protein score of $95 \%$ was considered highly reliable, those results were subject to a further bioinformatic analysis. Based on mass spectrum identification, 9 types of proteins were identified from the 49 significantly different protein spots. The protein score of each point was $100 \%$. Based on a comparison of the protein sequences and search in the NCBI non-redundant database, these were apolipoprotein A1 (APOA1), ACTG1, ALB, FGB, peroxiredoxin 2 (PRDX2), and FGG. The Mowse scores of the 6 proteins are presented in Fig. 1.

Western blot analysis verification of APOA1 and PRDX2. Actin served as the internal reference. The western blot analysis expression of APOA1 in the necrosis group and the control group is shown in Fig. 2. There was a significant difference in APOA1 protein content between the necrosis group and the control group as well as a significant difference in PRDX2 between the necrosis group and the control group (necrosis group $>$ control group, $\mathrm{P}<0.05$ ). 


\section{Discussion}

The recently developed fluorochrome marked two-dimensional electrophoresis technique, i.e., fluorescence DIGE (12) helps avoid the non-parallelism that occurs when different gels are used. Three samples marked with three-colour fluorochromes (Cy2, Cy3 and Cy5) can be electrophoresed on the same gel and the experiment has excellent credibility and repeatability. These fluorochromes can detect proteins of low abundance as they have a high sensitivity (13).

The causes of non-traumatic femoral head necrosis are complex; steroid-induced and alcohol-induced necrosis are the main causes (14). Pathogenesis of this disease still remains controversial. Differential proteomics is a major breakthrough of the proteomic research (15). It is intended to identify and screen differential protein by comparing the proteins expressed in the samples in different periods, different tissues, different states or under different external conditions. In our experiment, 49 significantly different proteins were successfully separated and identified. Based on the MALDITOF-MS identification technique (16) and bioinformatics analysis, it was found that PRDXs are the members of a recent PRDX family; they belong to the antioxidant protein family. These proteins are widely present in both procaryotic and eukaryotic organisms. They participate in multiple cellular functional activities including anti-oxidative damage, cell division, cellular differentiation, signal transduction, and cell apoptosis (17-21). The expression levels of PRDX II in the necrosis group was found to increase 10.68 times when compared to the control group. In the case of femoral head ischemia, the osteocytes suffered from hypoxia and ischemia. Thus, the number of the oxygen radicals were increased to stimulate increased expression of PRDX and produce the energy reduction in peroxides and superoxides. The energy reduction peroxides and superoxides were able to exert their antioxidant responses and eliminate oxygen free radicals; there were no studies on related research. The experiment also found that the expression levels of most protein spots in the fibrinogen chain increased. The increased expression levels may be on the interference of the residual blood during the washing treatment process. It may also be associated with the hypercoagulable state of the blood system in elderly patients. Another explanation may be that it is associated with the modification of proteins in the osseous tissue following femoral head necrosis. The expression of some protein spots is downregulated. Therefore, it can play a role in the regulation of the later necrosis repair period. The expression of ALB in the femoral head necrosis group is upregulated 6.8915.26 times when compared to those of the control group.

In combination with results from existing research on femoral head necrosis and the many pathogenesis doctrines [blood hypercoagulability state (22), lipid metabolism disorders (23), and metabolic disorders of oxygen-free radicals (24)], it can be inferred that the expression levels of most fibrinogens are upregulated in the advanced stage of femoral head necrosis due to blood hypercoagulability in the elderly patients. Lipid metabolism disorders, fat deposition in the femoral head, and the fat-occluded vessels lead to avascular necrosis. Bone repair occurs in the intermediate and advanced stages. Based on a negative feedback mechanism, the expres- sion of the apolipoprotein is most likely upregulated to remove excessive lipid deposition in the osseous tissue. Hypoxia and ischemia of the osseous tissue leads to metabolic disorders of oxygen-free radicals. Deposition leads to cellular injury and necrosis. The expression of transferrins is upregulated and excessive iron ions are transported, thus reducing the concentration of the free iron ions and preventing the toxic side effects of free iron on cells by stimulating the formation of free radicals. Increased expression of PRDX produces energy reduction peroxides and superoxides, thus allowing for formation of antioxidant response (25) and elimination of free radicals. Hypoxia and ischemia cause damage to the mitochondrial function, increased oxygen free radicals, and decrease the activity of the ATP synthetase (26). The ATP synthetase on the cytomembrane surface is highly ectopic (27) in the intermediate and advanced stages. It participates in the repair process through a certain negative feedback mechanism and signal transduction.

We should note that the sample size is small due to some constraints, which is a limitation of the study. The samples are taken from the necrotic femoral head during the hip joint replacement (intermediate and advanced stages) (Ficat $\mathrm{m} / \mathrm{IV}$ stage). If the osseous tissue samples of the femoral head were collected in the early stage of femoral head necrosis (Ficat I/II stage) for proteomic analysis, we could understand the dynamic changes in the protein of osseous tissue of the patients with femoral head necrosis and comprehensively discuss and analyze the pathogenesis of femoral head necrosis and provide the basis for early prevention and treatment.

\section{References}

1. Gagala J, Tarczyńska M and Gawęda K: Clinical and radiological outcomes of treatment of avascular necrosis of the femoral head using autologous osteochondral transfer (mosaicplasty): preliminary report. Int Orthop 37: 1239-1244, 2013.

2. Morales de Cano JJ, Gordo C and Illobre JM: Early clinical results of a new conservative hip stem. Eur J Orthop Surg Traumatol 24: 359-363, 2014.

3. Liu Y, Wu J, Zhu Y and Han J: Therapeutic application of mesenchymal stem cells in bone and joint diseases. Clin Exp Med 14: 13-24, 2014.

4. Ganz R, Slongo T, Turchetto L, Massè A, Whitehead D and Leunig M: The lesser trochanter as a cause of hip impingement: pathophysiology and treatment options. Hip Int 23 (Suppl 9): S35-S41, 2013.

5. Słowińska M, Kozłowski K, Jankowski J and Ciereszko A: Proteomic analysis of white and yellow seminal plasma in turkeys (Meleagris gallopavo). J Anim Sci 93: 2785-2795, 2015.

6. Çitlak A and Şener M: The incidence of avascular necrosis of the femoral head changes with the hip abduction angle in the hip spica in treatment of developmental dislocation of the hip. J Pediatr Orthop B 22: 608-609, 2013.

7. Sikulu MT, Monkman J, Dave KA, Hastie ML, Dale PE, Kitching RL, Killeen GF, Kay BH, Gorman JJ and Hugo LE: Proteomic changes occurring in the malaria mosquitoes Anopheles gambiae and Anopheles stephensi during aging. J Proteomics 126: 234-244, 2015.

8. Antonopoulos IH, Murayama Y, Warner BA, Sekine S, Yokoyama S and Carey PR: Time-resolved Raman and polyacrylamide gel electrophoresis observations of nucleotide incorporation and misincorporation in RNA within a bacterial RNA polymerase crystal. Biochemistry 54: 652-665, 2015.

9. Liao C, Peng ZY, Li JB, Cui XW, Zhang ZH, Malakar PK, Zhang WJ, Pan YJ and Zhao Y: Simultaneous construction of PCR-DGGE-based predictive models of Listeria monocytogenes and Vibrio parahaemolyticus on cooked shrimps. Lett Appl Microbiol 60: 210-216, 2015. 
10. Mimae T and Ito A: New challenges in pseudopodial proteomics by a laser-assisted cell etching technique. Biochim Biophys Acta 1854: 538-546, 2015.

11. Fan LQ, Li Y, Zhao Q, Tan BB, Ma LG, Liu Y and Wang D: Comparative proteomics in gastric cancer cell line BGC823 after ZNF139 gene inhibited with RNA interference. Hepatogastroenterology 61: 1822-1829, 2014.

12. Gok I, Baday M, Cetinkunar S, Kilic K and Bilgin BC: Polymorphisms in DNA repair genes XRCC2 and XRCC3 risk of gastric cancer in Turkey. Bosn J Basic Med Sci 14: 214-218, 2014

13. Kasempimolporn S, Lumlertdacha B, Chulasugandha P, Boonchang S and Sitprija V: Alterations in brain cerebral cortex proteome of rabies-infected cat. Southeast Asian J Trop Med Public Health 45: 808-815, 2014.

14. Mimae T, Ito A, Hagiyama M, Nakanishi J, Hosokawa Y, Okada M, Murakami Y and Kondo T: A novel approach to pseudopodia proteomics: excimer laser etching, two-dimensional difference gel electrophoresis, and confocal imaging. Protoc exch 2014: 2014.007, 2014.

15. Eskelinen AP, Moerland RJ, Kostiainen MA and Törmä P Self-assembled silver nanoparticles in a bow-tie antenna configuration. Small 10: 1057-1062, 2014.

16. Meng P, Hu W, Pei H, Hou Q and Ji Y: Effect of different plant species on nutrient removal and rhizospheric microorganisms distribution in horizontal-flow constructed wetlands. Environ Technol 35: 808-816, 2014.

17. Takahashi S, Fukushima W, Yamamoto T, Iwamoto Y, Kubo T, Sugano N and Hirota Y; Japanese Sentinel Monitoring Study Group for Idiopathic Osteonecrosis of the Femoral Head: Temporal trends in characteristics of newly diagnosed nontraumatic osteonecrosis of the femoral head from 1997 to 2011: a hospital-based sentinel monitoring system in Japan. J Epidemiol 25: 437-444, 2015.

18. Yang S, Halim AY, Werner BC, Gwathmey FW and Cui Q: Does osteonecrosis of the femoral head increase surgical and medical complication rates after total hip arthroplasty? A comprehensive analysis in the United States. Hip Int 25: 237-244, 2015.
19. Felden A, Vaz G, Kreps S, Anract P, Hamadouche M and Biau DJ: A cemented acetabular component with a reinforcement cross provides excellent medium-term fixation in total hip arthroplasty after pelvic irradiation. Bone Joint J 97-B: 177-184, 2015.

20. Yu PA, Peng KT, Huang TW, Hsu RW, Hsu WH and Lee MS: Injectable synthetic bone graft substitute combined with core decompression in the treatment of advanced osteonecrosis of the femoral head: a 5-year follow-up. Biomed J 38: 257-261, 2015.

21. Felten R, Messer L, Moreau P, Goussot R and Mahé A: Osteonecrosis of the femoral head linked to topical steroids for skin bleaching: a case report. Ann Intern Med 161: 763-764, 2014.

22. Crosby SN Jr, Kim EJ, Koehler DM, Rohmiller MT, Mencio GA, Green NE, Lovejoy SA, Schoenecker JG and Martus JE: Twenty-year experience with rigid intramedullary nailing of femoral shaft fractures in skeletally immature patients. J Bone Joint Surg Am 96: 1080-1089, 2014.

23. Murphey MD, Foreman KL, Klassen-Fischer MK, Fox MG, Chung EM and Kransdorf MJ: From the radiologic pathology archives imaging of osteonecrosis: radiologic-pathologic correlation. Radiographics 34: 1003-1028, 2014.

24. Zhang H, Xiao F, Liu Y, Zhao D, Shan Y and Jiang Y: A higher frequency of peripheral blood activated B cells in patients with non-traumatic osteonecrosis of the femoral head. Int Immunopharmacol 20: 95-100, 2014.

25. Tang XK, Ye FS, Tong PJ, Fan YH, Li M, Ying H and Xiao LW: Progress on tantalum rod implanting for the treatment of femur head necrosis. Zhongguo Gu Shang 26: 617-620, 2013 (In Chinese)

26. Parker M, Cawley S and Palial V: Internal fixation of intracapsular fractures of the hip using a dynamic locking plate: two-year follow-up of 320 patients. Bone Joint J 95-B: 1402-1405, 2013.

27. Li J, Li Y, Wang Y, Liu M and Zhao G: Preventive effects of siRNA targeting PPAR $\gamma$ gene on steroid-induced osteonecrosis in rabbits. Connect Tissue Res 55: 322-330, 2014. 